\title{
COLLISIONS BETWEEN TWO PHASE-INVERSION-WAVES IN AN ARRAY OF OSCILLATORS
}

\author{
Masayuki YAMAUCHI, Masahiro WADA, Y oshifumi NISHIOand Akio USHIDA \\ Dept. of Electrical and Electronic Engineering, Tokushima Universit y, Japan \\ \{masa,wada,nishio,ushida\}@ee.tokushima-u.ac.jp
}

\begin{abstract}
In this study, the phenomena related to collisions betw een $t \mathrm{w}$ o phase-inersion-waves in an array of van der $\mathrm{P}$ ol oscillators are investigated. Behavior of the tw o phase-in ersion-waves after they collide with ead other is classified by computer simulations. F urther, the mechanisms of the complete extinction and reflection of the two waves b y using the relationship between phase states and oscillation frequencies are explained.
\end{abstract}

\section{Introduction}

A lot of studies on synchronization phenomena of coupled oscillators ha vebeen carried out up to now. Endo et al. have reported a details of theoretical analysis and circuit experiments about some coupled oscillators as a ladder, a ring and a tw o-dimensional arra [1][3]. Recently, wave propagation phenomenon observed from coupled chaotic circuits is also reported [4][5]. How ever,such studies treat only transient states for a giv en set of initial conditions and there seems to be very few studies on contin uously existing wave propagation phenomenon observed simple coupled oscillators circuits.

On the other hand, w eha vereported in our past study [6] wave propagation phenomena of phase states in van der Pol oscillators coupled by inductors as a ladder. In that study, w efound "phase-inversion-wa ve" which is continuously existing wave of changing phase states between tw oadjacent oscillators from in-phase to an ti-phase or an ti-phase to in-phase. F urther, w e explained the mechanisms of the propagation and the reflection at the edge of the ladder by using the relationship betw een phase states and oscillation frequency

In this study, we pay our attentions on the phenomena related to collisions between tw o phase-inersion$\mathrm{w}$ aes in the same circuit. We can confirm by computer simulations that the behavior of the tw o phaseinversion-waves after they collide with eac h other is classified into three types; completely extinction, completely reflection and intermediate complex phenomenon. F urther, w ecan explain the mechanisms of the completely extinction and the completely reflection of the two waves by using the relationship betw een phase states and oscillation frequency.

\section{Circuit Model}

Circuit model is shown in Fig. $1 . N$ van der $\mathrm{P}$ ol oscillators are coupled by coupling inductors $L_{0}$. We carry out computer calculations for the case of $N=$ 17. In the computer calculations, we assume the $v-$ $i$ characteristics of nonlinear negative resistors in the circuit by the following functions.

$$
i_{r}\left(v_{k}\right)=-g_{0} v_{k}+g_{3} v_{k}^{3} \quad\left(g_{1}, g_{3}>0\right) .
$$

The circuit equations governing the circuit in Fig. 1 are expressed as

$$
\begin{aligned}
& \dot{x}_{k}=y_{k} \\
& \dot{y}_{k}=-x_{k}+\alpha\left(x_{k+1}-2 x_{k}+x_{k-1}\right)+\varepsilon\left(y_{k}-\frac{1}{3} y_{k}^{3}\right) \\
& \left(k=1,2, \cdots, N, \quad x_{0}=x_{1}, \quad x_{N+1}=x_{N}\right)
\end{aligned}
$$

where

$$
\begin{gathered}
t=\sqrt{L_{1} C} \tau, i_{L_{1} k}=\sqrt{\frac{C g_{1}}{3 L_{1} g_{3}}} x_{k}, v_{k}=\sqrt{\frac{g_{1}}{3 g_{3}}} y_{k}, \\
\alpha=\frac{L_{1}}{L_{0}}, \varepsilon=g_{1} \sqrt{\frac{L_{1}}{C}}, \frac{d}{d \tau}=" . "
\end{gathered}
$$

It should be noted that $\alpha$ corresponds to the coupling and that $\varepsilon$ corresponds to the nonlinearity. Equations (2) are calculated by using the fourth-order Runge-Kutta method.
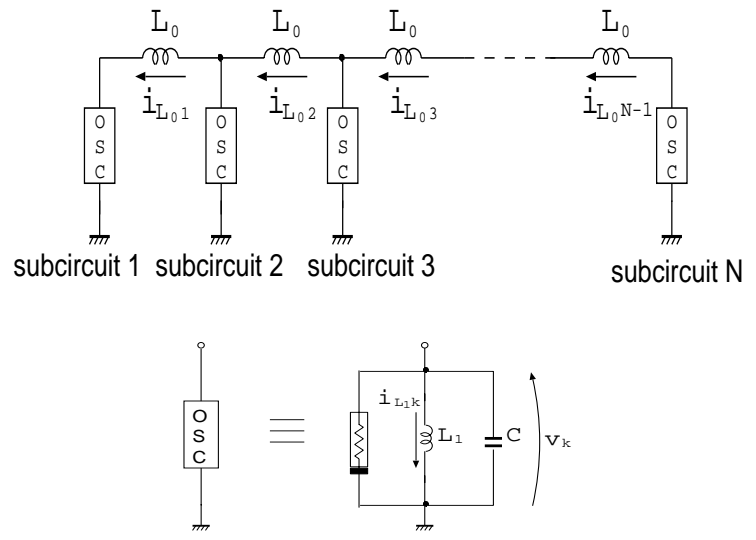

Figure 1: Array of van der Pol oscillators.

Figure 2 shows a typical example of observed phaseinversion-wa ves. In upper diagrams, vertical axes are sum of voltages of adjacent oscillators and horizontal axes are time. Hence, the diagrams show ho w phase differences betw een adjacent oscillators change as time goes. White regions in the diagram correspond to the states that tw o adjacent oscillators are anti-phase synchronization. While, black regions correspond to the in-phase synchronization. In lower figures, snapshots of attractor of each oscillator and phase states betw een adjacent oscillators are shown.

In our previous study [6], we have investigated the propagation and the reflection at the edge of the ladder of the wave. 


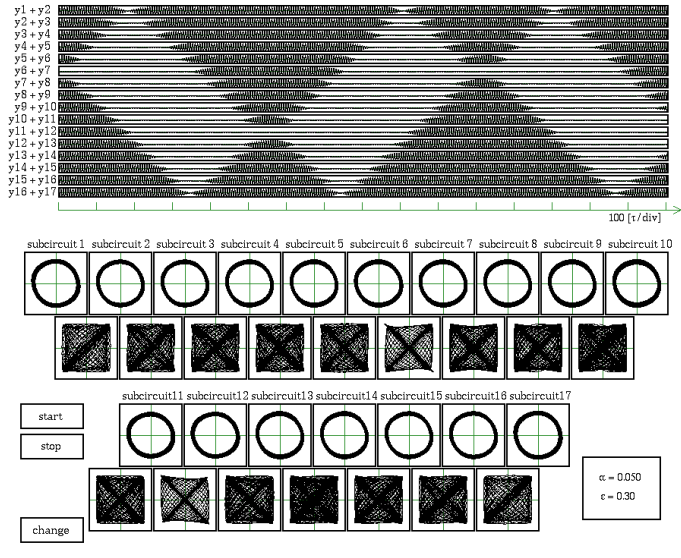

Figure 2: T ypical example of phase-inversion$\mathrm{w}$ a es.

Remark: Wave propagation phenomenon seems to be observed for an y number of coupled oscillators. We could observe similar phenomenon from both of even and odd numbers of oscillators and from both of small (5) and large (100) numbers.

\section{Collision between Two Phase- Inv ersion- Wá $v$ es}

We can see in Fig. 2 that the tw o phase-inersionw a completely reflect when they collide with eac $h$ other. Howev er, by giving different initial conditions w e can also obsere that they completely extinct after the collision (see Fig. 3).

In order to make clear the condition of the generation of the tw ocompletely different phenomena, w e investigate the mechanisms of these phenomena by using the relationship betw een phasestates and oscillation frequency. It has been already known that oscillation frequency of in-phase synchronization of oscillators coupled by inductors is different from that of anti-phase synchronization. Namely, $f_{i n}$, oscillation frequency of in-phase synchronization, is smaller than $f_{a n t i}$, oscillation frequency of anti-phase synchronization. F urther, the difference betw eens $f_{i n}$ and $f_{\text {anti } i}$ increases as coupling inductance increases [7].

Throughout the paper, we define the phase difference betw een $\mathrm{t} \mathrm{w}$ o adjaceroscillators and the frequency of OSC $k$ as follo ws:

$$
\begin{aligned}
& \Phi_{k, k+1}(n)=\frac{\tau_{k}(n)-\tau_{k+1}(n)}{\tau_{k}(n)-\tau_{k}(n-1)} \times \pi \\
& f_{k}(n)=\frac{1}{2\left(\tau_{k}(n)-\tau_{k}(n-1)\right)}
\end{aligned}
$$

where $\tau_{k}(n)$ is time when the voltage of OSC $k$ crosses $0[\mathrm{~V}]$ at $n$-th time.

Remark: The $f_{k}(n)$ in Eq. (4) should be called zerocrossing-time instead of frequency. However, since $f_{k}(n)$ must be compared with $f_{\text {in }}$ and $f_{a n t i}$, which is oscillation frequency observed from the two coupled oscillators, we use the term frequency.

\subsection{Mechanism of complete extinction}

1. Let us assume that OSC $5 \sim \mathrm{OSC} 7$ are anti-phase synchronization and that the wa ves changing from an ti-phase into in-phase are going to reach OSC5 and OSC7 at the almost same time from the directions of OSC1 and OSC17 respectively.

2. Reaching the w a causes the changes of oscillation frequencies of OSC5 $f_{5}$ and OSC $7 f_{7}$ changes from $f_{\text {anti } i}$ to $f_{\text {in }}$.

3. Accordingly $\Phi_{5,6}$ and $\Phi_{6,7}$ change from $\pi$ to 0 and $2 \pi$ respectively.

4. Changes of $\Phi_{5,6}$ and $\Phi_{6,7}$ cause that the change of oscillation frequency of OSC $6 f_{6}$ from $f_{a n t i}$ to $f_{\text {in }}$.

5. When $\Phi_{5,6}$ and $\Phi_{6,7}$ reach $2 \pi$ and 0 respectively, $f_{6}$ become to be equal to $f_{i n}$. The whodera y results in stable in-phase synchronization.

Wa ves changing from in-phase to anti-phase can be explained in a similar manner.

Figure 3 sho ws computer calculated results corresponding to the completely extinction. We can see a $\mathrm{w}$ ave extinction occurs as the manner explained abve.

\subsection{Mechanism of complete reflection}

1. Let us assume that OSC7 $\sim$ OSC10 are in-phase synchronization and that the wa ves changing from in-phase into an ti-phase are going to read OSC7 and OSC10 at the almost same time from the directions of OSC1 and OSC17, respectively.

2. Waves reach OSC7 and OSC10 almost equal timing. $\Phi_{7,8}$ and $\Phi_{9,10}$ approach $\pi$ and $-\pi$ by almost equal timing.

3. Because $\Phi_{7,8}$ and $\Phi_{9,10}$ approach $\pi$ and $-\pi$ respectively, oscillation frequencies of $\mathrm{OSC}_{8} f_{8}$ and OSC9 $f_{9}$ change from $f_{\text {in }}$ tow ard $f_{\text {anti }}$.

4. How ev er,because $f_{8}$ and $f_{9}$ change almost simultaneously, in-phase synchronization betw een OSC 8 and OSC 9 does not break. Namely, $\Phi_{8,9}$ remains almost 0 . Hence, $f_{8}$ and $f_{9}$ do not reach $f_{\text {anti. }}$. Accordingly $\Phi_{7,8}$ and $\Phi_{9,10}$ continue to change until reac hing $2 \pi$ and $-2 \pi$, respectively .

5. By the effect of the decreases of $\left(\Phi_{7,8} \bmod 2 \pi\right)$ and $\left(\Phi_{9,10} \bmod 2 \pi\right), f_{8}$ and $f_{9}$ begin to decrease toward $f_{\text {in }}$ again.

Wa ves changing from anti-phase to in-phase can be explained in a similar manner.

Figure 4 sho ws computer calculated results corresponding to the completely reflection. We can see wave reflections occur as the manner explained above.

According to the abo ve-explainedmechanisms of the completely extinction and the completely reflection, w ecan conclude that the tw ophase-inversion$\mathrm{w}$ aves colliding ealr other will; 

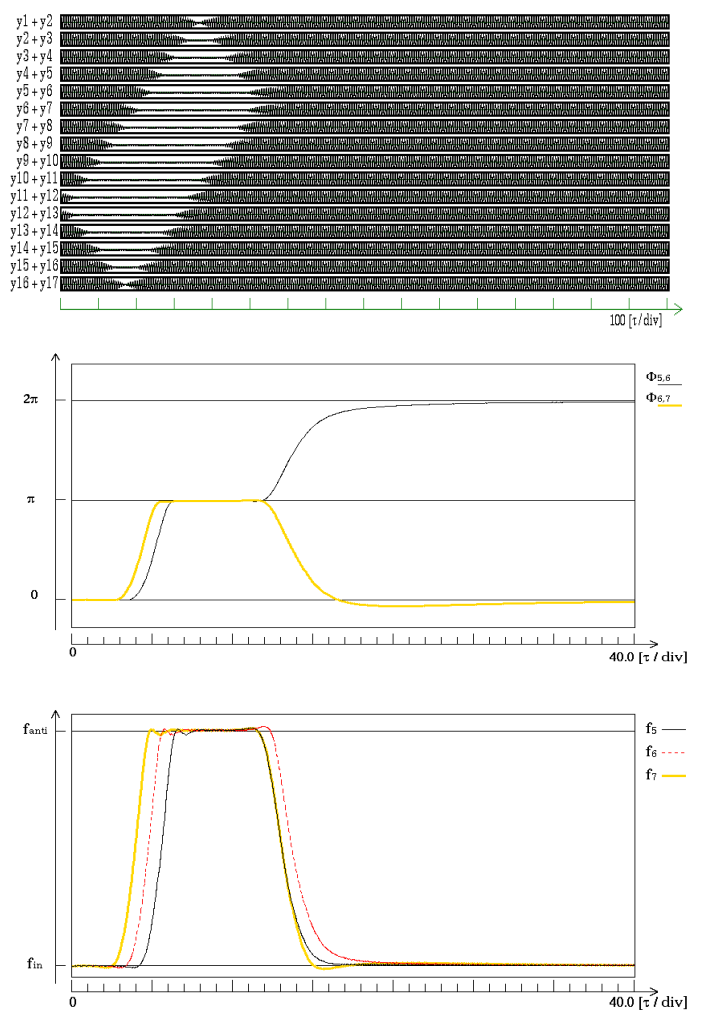

Figure 3: Completely extinction.

1. Completely reflect when the w a reach OSCk and OSC $k+1$ at the almost equal timing.

2. Completely extinct when the waves read OSC $k-$ 1 and $\mathrm{OSC} k+1$ at the almost equal timing.

\subsection{Between complete extinction and complete reflection}

From the abo veconclusion, our next question is what kind of phenomena will be generated when the tw o phase-inversion-waves collide each other with time difference.

In order to investigate the in termediate phenomena, we generate tw o phase-in ersion-wa ves from both edges of the arra y with time delay for one side. $T_{i n}$ is the period of oscillation frequency of the in-phase synchronization $\left(T_{i n}=1 / f_{i n}\right)$.

Obtained computer simulated results from various values of the time delay are shown in Fig. 5.

1. Figure 5(a): After the collision, tw o phase-in ersion$\mathrm{w}$ aves completely extinct.

2. Figure 5(b): After the collision, small lev el of phase difference remains. The phase difference propagate to one direction. It is atten uated as time goes and extinct soon. This phenomenon are observed for the time delay smaller than 0.9 $T_{\text {in }}[\tau]$.

3. Figure 5(c): After the collision, a relatively large
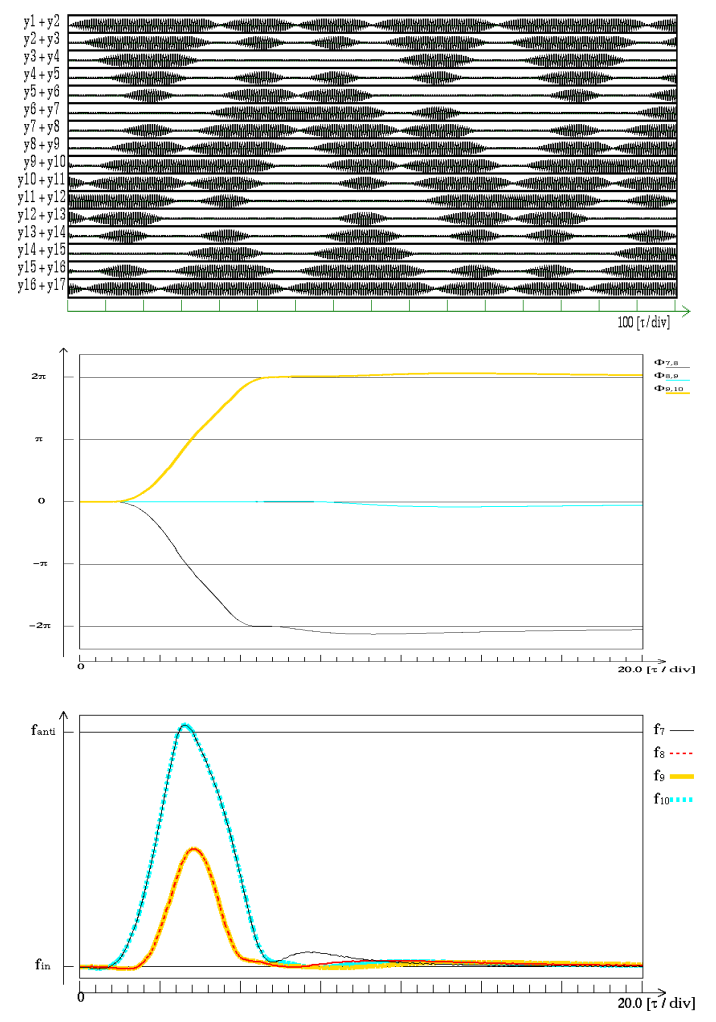

Figure 4: Completely reflection.

lev el of phase difference remains. The phase difference grows to $\pi$ and propagates as a phaseinversion-wave.

4. Figures 5(d) (f): Complex wa ve propagation phenomenon is observed. $\mathrm{V}$ arious wa $\mathrm{v}$ es of different level of phase differences are produced after the collision and they influence each other.

5. Figure 5(g)After the collision, $\mathrm{t}$ w o phase-in ersion$\mathrm{w}$ a es completely reflect. This phenomenon are observed for the time delay larger than $1.7 T_{i n}[\tau]$.

Figure 6 shows the relationship betw een time dela and observed phenomena. Because the time that the phase-inversion-wave propagate from one oscillator to the next is about $5 T_{i n}[\tau]$, the structure of this figure will repeat as time delay increases. F urther, ne can say that the reflection can be generated more easily than extinction.

\section{Conclusions}

In this study w einvestigated the phenomena related to collisions betw een tw ophase-inversion-waves in an array of van der $\mathrm{P}$ ol oscillators. We coud confirm by computer simulations that the behavior of the two phase-inversion-waves after they collide with each other was classified into three types; completely extinction, completely reflection and in termediate complex 


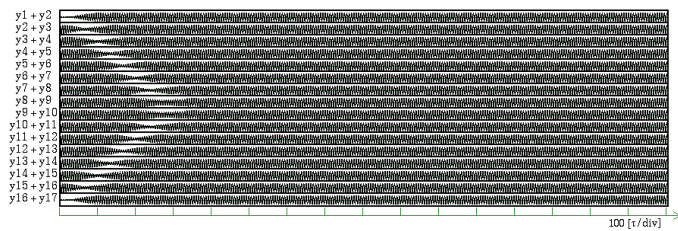

(a) delay $=0.00 T_{\text {in }}[\tau]$.

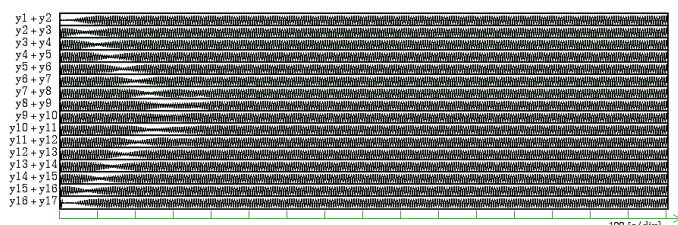

(b) delay $=1.50 T_{i n}[\tau]$.

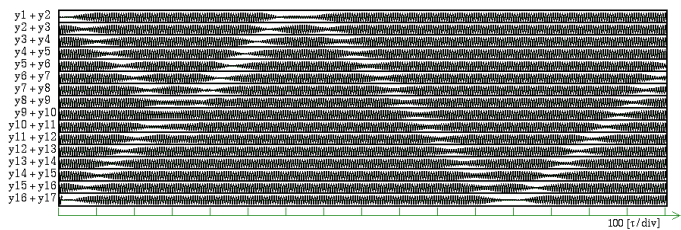

(c) dela $\mathrm{y}=100 T_{i n}[\tau]$.

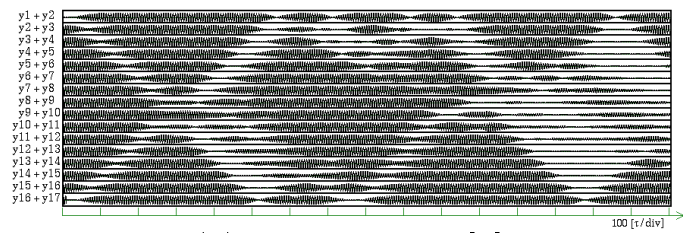

(d) delay $=1.25 T_{i n}[\tau]$.

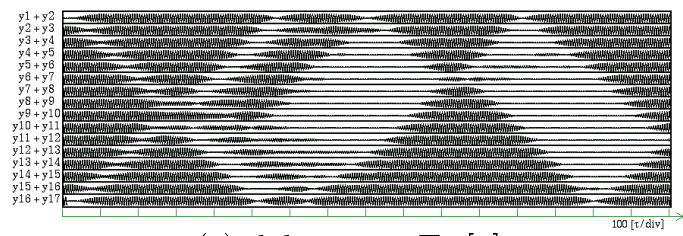

(e) dela $\mathrm{y}=150 T_{i n}[\tau]$.

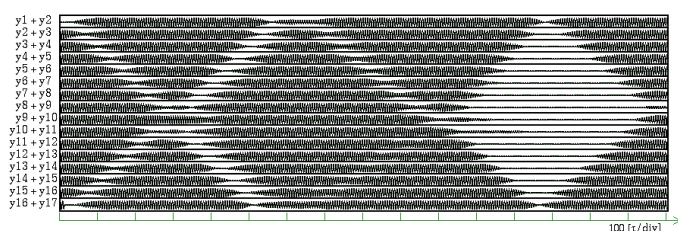

(f) delay $=1.65 T_{i n}[\tau]$.

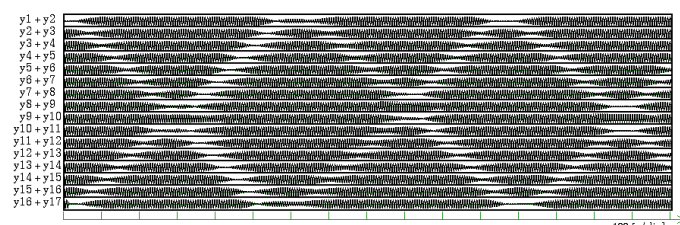

(g) delay $=1.70 T_{\text {in }}[\tau]$.

Figure 5: Change from complete extinction to complete reflection.

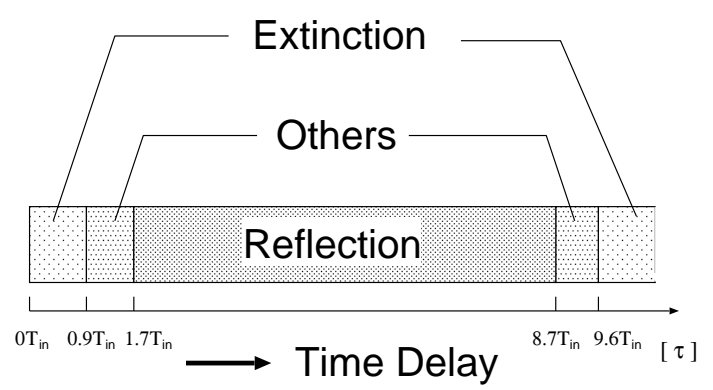

Figure 6: Domain of extinction, reflection and other phenomena.

phenomenon. F urther, w ecould explain the mechanisms of the completely extinction and the completely reflection of the tw ow a bs bysing the relationship betw een phase states and oscillation frequency

\section{REFERENCES}

[1] T. Endo and S. Mori "Mode Analysis of a Multimode Ladder Oscillator," IEEE T xans. Circuits Syst., vol. 23, pp. 100-113, Feb. 1976.

[2] T. Endo and S. Mori, "Mode Analysis of TwoDimensional Low-Pass Multimode Oscillator," IEEE T xans. Circuits Syst., vol. 23, pp. 517-530, Sep. 1976.

[3] T. Endo and S. Mori, "Mode Analysis of a Ring of a Large Number of Mutually Coupled van der Pol Oscillators," IEEE Trans. Circuits Syst., vol. 25, pp. 7-18, Jan. 1978.

[4] M. J. Ogorzalek, Z. Galias, A. M. Dabrowski and W. R. Dabrowski, "Chaotic Waves and SpatioT emporal Patterns in Large Arrays of Doubly-Coupled Ch ua's Circuits,"IEEE Trans. Circuits Syst. I, vol. 42, pp. 706-714, Oct. 1995.

[5] L. Pivka, "Autow aves and Spatio- Tmporal Chaos in CNNs-Part I: A Tutorial," IEEE Trans. Circuits Syst. I, vol. 42, pp. 638-649, Oct. 1995.

[6] M. Yamauchi, M. Wada, Y. Nishio and A. Ushida, "Wave Propagation Phenomena of Phase States in Coupled Oscillators," $\operatorname{Pr}$ ๔. of ECCTD'99, vol. 2, pp.695-698, Aug. 1999.

[7] T. Suezaki and S. Mori, "Mutual Synchronization of Two Oscillators," Trans of IECE, vol. 48, no. 9, pp. 1551-1557, Sep. 1965. 